\title{
Guided Waves Propagation in Two-layer Tubes: Effects of the Inner Part in Contact with Air in the Cavity
}

\author{
Abdelkader Elhanaoui \\ LMTI, Ibn Zohr University \\ Faculty of Sciences \\ BP 8106 Agadir 80000, \\ Morocco
}

\author{
Elhoucein Aassif \\ LMTI, Ibn Zohr University \\ Faculty of Sciences \\ BP 8106 Agadir 80000, \\ Morocco
}

\author{
Dominique Décultot \\ LOMC UMR CNRS 6294, \\ Normandie Université \\ 75 rue Bellot, CS 80 540, \\ Le Havre University, \\ 76058 Le Havre,
}

\author{
Gérard Maze \\ LOMC UMR CNRS 6294, \\ Normandie Université \\ 75 rue Bellot, CS 80 540, \\ Le Havre University \\ 76058 Le Havre, \\ France
}

\begin{abstract}
This paper studies the acoustic backscattering of short ultrasonic pulses by air-filled stainless steel-solid polymer two-layer cylindrical tubes immersed in water. The stainless steel one-layer tube is taken as a reference. The focus of this paper is on revealing the effects of physical characteristics of the solid polymer on the scattering phenomenon. The work is done from the calculation of the backscattered pressure, an inverse Fourier Transform, which provides a temporal signal. Wigner-Ville representation has been chosen in order to analyze the acoustic signal backscattered by each tube. For reduced frequencies ranging from 0.1 to 200 , the resonance spectrum and resonance trajectories have shown the manifestations of the guided waves. In this respect, the bifurcation of the $\boldsymbol{A}_{\boldsymbol{0}}$ wave to the $\boldsymbol{A}_{\boldsymbol{0}}{ }^{-}$and the $\boldsymbol{A}_{\boldsymbol{0}}{ }^{+}$waves has been observed. The authors investigate the reduced cutoff frequencies of the symmetrical and the antisymmetrical guided waves, specially the curves changes. The findings are then compared with those obtained for the stainless steel onelayer cylindrical tube. Reduced cutoff frequency values have also been extracted from Wigner-Ville time-frequency images. A good agreement has, therefore, been obtained. The study of acoustic scattering by stainless steel-solid polymer two-layer tubes has revealed the sliding of the reduced cutoff frequencies of $\boldsymbol{A}_{1}$ and $\boldsymbol{S}_{1}$ guided waves towards low values, due the repulsion phenomena. The relationship between reduced cutoff frequency and velocity of wave in the solid polymer is linear; which is a very interesting result.
\end{abstract}

\section{Keywords}

Acoustic backscattering; Two-layer cylindrical tube; Resonance spectrum; Wigner-Ville time-frequency representation.

\section{INTRODUCTION}

The acoustic scattering of a short impulse by elastic solids has been theoretically formulated by $\mathrm{H}$. Überall and his collaborators [1,2]. The results of Resonant Scattering Theory (R.S.T.) reveal that the resonances of an air-filled shell immersed in water are connected to the propagation of circumferential or peripheral waves. The
Method of Isolation and Identification of Resonances (M.I.I.R.) experimentally justifies the (R.S.T.) [3, 4]. Consequently, the backscattering spectrum, the resonance spectrum and the modes of the vibration can be determined. The studies of submerged air-filled cylindrical and spherical shells have shown, in the low frequency range, the bifurcation of the dispersion curve for the circumferential antisymmetrical Lamb $\boldsymbol{A}_{0}$ wave [5-7]. The authors of these references suggested a repulsion phenomenon in the phase velocity dispersion curve of the circumferential $\boldsymbol{A}_{0}$ wave when the cylindrical shell is in contact with the outer fluid. The corresponding waves were denoted by $\boldsymbol{A}_{0}{ }^{-}$and $\boldsymbol{A}_{0}{ }^{+}$. Other works are focused on the acoustic scattering from cylindrical shells filled with water or a polymer coupled by a thin layer of water $[8,9]$. Resonances of the guided waves were experimentally examined. While work studies on acoustic scattering from one-layer structures are frequent in the literature, very few investigated acoustic scattering from multi-layer cylindrical tubes. Nevertheless, few studies have examined the acoustic scattering from functionally graded cylindrical shells [10-13]. The paper provides the study of several acoustic signals backscattered by air-filled stainless steel-solid polymer two-layer tubes immersed in water. WignerVille $(W V)$ representation is a powerful tool and has been utilized in the applications [18-20]. For the reduced frequencies ranging from 0.1 to 200 , the acoustic signals analysis has been performed for the stainless steel onelayer and the stainless steel-solid polymer two-layer tubes. The backscattering involves the propagation of various types of guided waves. Evaluations and comparisons made on resonance trajectories and timefrequency images show the sliding of the reduced cutoff frequencies of $\boldsymbol{A}_{1}$ and $\boldsymbol{S}_{1}$ guided waves towards low values. The interaction of guided waves is also manifested in the backscattering phenomenon.

\section{FORM FUNCTION}

\subsection{Theoretical study}

Figure 1 shows a plane acoustic wave of angular frequency $\omega$ normally incident on a submerged cylindrical two-layer tube 
of infinite length, outer radius $\boldsymbol{a}_{1}$, intermediary radius $\boldsymbol{a}_{2}$, and inner radius $\boldsymbol{a}_{3} .(\boldsymbol{x}, \boldsymbol{y}, \boldsymbol{z})$ is the Cartesian coordinate system with origin at $O$, the $z$ direction is coincident with the axis of the cylindrical tube. The corresponding cylindrical polar coordinate system $(\boldsymbol{r}, \boldsymbol{\theta})$ is chosen.

The incident acoustic pressure of the incident plane wave $\boldsymbol{p}_{\text {inc }}(\boldsymbol{r}, \boldsymbol{\theta})$ can be written as follows [14,15],

$$
p_{\text {inc }}(r, \theta)=P_{0} \sum_{n=0}^{\infty}\left[\varepsilon_{n} i^{n} J_{n}\left(k_{\text {water }} r\right)\right] \cos (n \theta)
$$

Where $\boldsymbol{k}_{\text {water }}=\boldsymbol{\omega} / \boldsymbol{C}_{\text {water }}$ is the wave number in the fluid medium outside the tube (medium $1=$ water), the sound has a velocity in water $\boldsymbol{C}_{\text {water }}$ equal to $1500 \mathrm{~m} \cdot \mathrm{s}^{-1}, \boldsymbol{\varepsilon}_{\boldsymbol{n}}$ the Neumann factor $\left(\varepsilon_{n}=2-\delta_{n 0}\right), i^{2}=-1, P_{0}$ the incident plane wave amplitude, and $\boldsymbol{J}_{n}$ are the cylindrical Bessel functions of the first kind of order $\boldsymbol{n}$ [16].

The scattered wave pressure $\boldsymbol{p}_{\text {scat }}$ at point $\boldsymbol{M}$ must be symmetrical about $\theta=0$ and, therefore, of the form,

$$
p_{\text {scatt }}(\omega)=P_{0} \sum_{n=0}^{\infty}\left[\varepsilon_{n} \frac{D_{n}^{(1)}(\omega)}{D_{n}(\omega)} i^{n} H_{n}^{(1)}\left(k_{\text {water }} r\right)\right] \cos (n \theta) \square \square
$$

Where $\boldsymbol{H}_{\boldsymbol{n}}{ }^{(l)}$ are the Hankel functions of the first kind of order $n, D_{n}^{(I)}(\omega)$ and $D_{n}(\omega)$ the determinants [16]. By applying the boundary conditions of the problem (continuity of stress and displacement at the interfaces), the terms of these determinants are determined at any given value of reduced frequency $\boldsymbol{x}_{\boldsymbol{I}}=\boldsymbol{k}_{\text {water }} \boldsymbol{a}_{\boldsymbol{l}}$.

The backscattered pressure in the far-field (form function $\left.F_{\infty}\right)$ is obtained from the following equation [17],

$$
F_{\infty}\left(x_{1}, \pi\right) \approx \frac{2}{\sqrt{\pi x_{1}}}\left|\sum_{n=0}^{\infty}(-1)^{n} \varepsilon_{n} \frac{D_{n}^{(1)}(\omega)}{D_{n}(\omega)}\right|
$$

\subsection{Form functions and Impulse responses}

Five cylindrical tubes comprising an outer layer of stainless steel in contact with water (fluid medium 1) and a layer of stainless steel or a solid polymer on the inner part in contact with air (fluid medium 2) in the cavity, are considered. These tubes are characterized by the same geometric properties; the outer radius of each structure is $\boldsymbol{a}_{1}=1 \mathrm{~m}$, the intermediary radius is $\boldsymbol{a}_{2}=0.96 \mathrm{~m}$, and its inner radius is $\boldsymbol{a}_{3}=0,94 \mathrm{~m}$. Thicknesses of the inner and outer parts are respectively $\boldsymbol{a}_{2}$ $\boldsymbol{a}_{3}=2 \mathrm{~cm}$ and $\boldsymbol{a}_{1}-\boldsymbol{a}_{2}=4 \mathrm{~cm}$. Physical properties of the studied tubes are given in Table 1. Backscattering spectrum of each tube is calculated for reduced frequencies ranging from 0.1 to 200. (See Fig. 2). In the calculations, the bandwidth of the transducer centered on the reduced frequency $x=100$ is considered.

Table 1. Physical characteristics

\begin{tabular}{|c|l|l|l|l|l|}
\hline $\begin{array}{c}\text { Tube } \\
\text { number }\end{array}$ & $\mathbf{0}$ & $\mathbf{1}$ & $\mathbf{2}$ & $\mathbf{3}$ & $\mathbf{4}$ \\
\hline Inner part & $\begin{array}{l}\text { Stainle } \\
\text { ss } \\
\text { steel }\end{array}$ & $\begin{array}{l}\text { Solid } \\
\text { polym } \\
\text { er 1 }\end{array}$ & $\begin{array}{l}\text { Solid } \\
\text { polym } \\
\text { er 2 }\end{array}$ & $\begin{array}{l}\text { Solid } \\
\text { polym } \\
\text { er 3 }\end{array}$ & $\begin{array}{l}\text { Solid } \\
\text { polym } \\
\text { er 4 }\end{array}$ \\
\hline
\end{tabular}

\begin{tabular}{|c|l|l|l|l|l|}
\hline $\begin{array}{c}\text { Density } \\
\left(\mathrm{kg} \cdot \mathrm{m}^{-3}\right)\end{array}$ & 7900 & 1500 & 1400 & 1100 & 900 \\
\hline $\begin{array}{c}\text { Longitudin } \\
\text { al } \\
\text { velocity } \\
\left(\mathrm{m} \cdot \mathrm{s}^{-1}\right)\end{array}$ & 5790 & 2500 & 2200 & 1800 & 1500 \\
\hline $\begin{array}{c}\text { Transversa } \\
1 \\
\text { velocity } \\
\left(\mathrm{m} \cdot \mathrm{s}^{-1}\right)\end{array}$ & 3100 & 1200 & 1100 & 900 & 700 \\
\hline
\end{tabular}

Many amplitude variations due to the presence of various resonances are observed on the form functions (Fig. 2).

For each tube, the total time signal is obtained from the form function by applying an inverse Fourier transform. To obtain the resonance spectrum, the specular echo is suppressed and replaced by zeros. Figure 3 presents the filtered signals for the studied shells.

\section{WIGNER-VILLE (WV)}

Time-frequency representations $(\boldsymbol{T F R})$ are important to analyze non-stationary signals $[18,19] . \boldsymbol{W} \boldsymbol{V}$ representation is chosen for the study of acoustic signals backscattered by airfilled cylindrical tubes immersed in water; specially that it ensures the best resolution. In plus, its windowed version reduces cross-terms that are a serious problem [20]. $W V$ representation is defined by [20],

$$
W V_{s}(t, v)=\int_{-\infty}^{+\infty} s\left(t+\frac{\tau}{2}\right) s^{*}\left(t-\frac{\tau}{2}\right) e^{-i 2 \pi v \tau} d \tau
$$

Where $s$ is the signal needed to analyze, $s^{*}$ its complex conjugate .Time and frequency are denoted by $\boldsymbol{t}$ and $\boldsymbol{v}$. expressed as:

$$
\text { Smoothed pseudo Wigner-Ville (SPWV) is }
$$

$\operatorname{SPWV}(t, v)=$

$\int_{-\infty}^{+\infty}\left|h\left(\frac{\tau}{2}\right)\right|^{2} \int_{-\infty}^{+\infty} \int g(t-\eta) s\left(\eta+\frac{\tau}{2}\right) s^{*}\left(\eta-\frac{\tau}{2}\right) e^{-i 2 \pi v \tau} d \eta d \tau$

Windowing with $\boldsymbol{h}$ and $\boldsymbol{g}$ reduces cross-terms but at the cost of losing some resolution.

The version of Eq. (5) for a discrete-time analytic signal $\boldsymbol{s}_{\boldsymbol{A}}(\boldsymbol{n})$ is:

$$
\begin{aligned}
& \operatorname{SPWV}(t, v)=2 \sum_{n=0}^{2 N-1} \sum_{m=-M+1}^{M+1}|h(n)|^{2} g(m) s_{A}(t+m+n) \cdot s_{A}^{*}(t+m-n) \\
& . \exp (-i 4 \pi v n)
\end{aligned}
$$

\section{RESULTS AND DISCUSSIONS}

\subsection{Resonance spectrums}

By applying the Fourier transform to each signal of Fig. 3, the resonance spectrum is obtained. Resonance series are presented in Fig. 4. 


\subsection{Resonance trajectories}

To study the effects of the inner part on the scattering phenomenon, the resonance spectrums are calculated for each mode $\mathrm{n}$. Then, the assemblages are determined. The resonance trajectories for the one-layer stainless steel tube and the twolayer stainless steel -solid polymer tubes are presented in the same graphic; see Fig. 5.

In Figure 5, fine resonances appear as points while broad resonances turn out to be substantially continuous and dark.

\subsection{Effects of the inner part and comparison}

From Figure 5, the reduced cutoff frequencies of the $\boldsymbol{A}_{\boldsymbol{I}}$ and $\mathrm{S}_{\boldsymbol{I}}$ pseudo-waves corresponding to each tube are determined with a relatively good precision. The results are summarized in Table 2.

Table 2. Obtained reduced cutoff frequencies by resonance trajectories $\left(x_{I c}\right)$

\begin{tabular}{|l|l|l|l|l|l|}
\hline Tube number & $\mathbf{0}$ & $\mathbf{1}$ & $\mathbf{2}$ & $\mathbf{3}$ & $\mathbf{4}$ \\
\hline $\boldsymbol{x}_{I c}\left(\boldsymbol{A}_{\boldsymbol{I}}\right)$ & 110.7 & 65.6 & 61.2 & 49.7 & 38.7 \\
\hline $\boldsymbol{x}_{I c}\left(\boldsymbol{S}_{\boldsymbol{I}}\right)$ & $\ldots$ & 135.2 & 119.6 & 98 & 81.2 \\
\hline
\end{tabular}

The findings indicate that the reduced cutoff frequencies of $\boldsymbol{A}_{\boldsymbol{I}}$ and $S_{1}$ pseudo-waves have slide towards low values. In addition, the shape of the trajectories shows the repulsion phenomena due to the interaction of various guided waves.

The comparison of different resonance trajectories leads us to a number of remarks:

- The trajectory assigned to the $\boldsymbol{A}_{\boldsymbol{0}}$ wave (stainless steel tube placed in vacuum), is separated into $\boldsymbol{A}_{\boldsymbol{0}}{ }^{-}$ and $\boldsymbol{A}_{0}{ }^{+}$trajectories. Indeed, all tubes are immersed in water.

- The external propagation of the $\boldsymbol{A}_{\boldsymbol{0}}{ }^{-}$wave around each tube confirms the insensitivity of the tube's inner part.

- However, it should be noted that the $\boldsymbol{A}_{0}{ }^{+}$pseudowave trajectory is sensitive to the physical properties of the tube's inner part. For higher modes (60-80), a gradual deviation towards lower frequencies and possibly attenuation are noted.

- When the physical characteristics of the inner part decrease, The $\boldsymbol{S}_{\boldsymbol{0}}$ pseudo-wave trajectory is identical to the trajectory of the $\boldsymbol{A}_{\boldsymbol{0}}{ }^{+}$pseudo-wave for $\boldsymbol{n}$ about 50 . Then, for the higher modes, a deflection toward the low frequency is observed.

- The trajectory of the $\boldsymbol{A}_{\boldsymbol{I}}$ wave (Fig.5.a) for the stainless steel one-layer tube has a reduced cutoff frequency around the value 110.7. The trajectories of the $\boldsymbol{A}_{\boldsymbol{I}}$ wave (Fig.5.b to Fig.5.e) for the air-filled stainless steel-solid polymer two-layer tubes are significantly different. It curves towards lower frequencies. In this respect, it should be noted that the reduced cutoff frequency is decreased as velocity of the transversal wave in the solid polymer.
- The trajectory of the $S_{1}$ wave (Fig.5.b) has a reduced cutoff frequency equal to 135.2. This reduced cutoff frequency decreases as velocity of the longitudinal wave in the solid polymer (Fig.5.c to Fig.5.e).

Figure 6 shows the evolution of reduced cutoff frequencies of the $A_{1}$ and the $S_{1}$ waves as a function respectively of the transversal and the longitudinal velocities in the solid polymer.

The obtained relation is as follows,

$$
x_{1 \mathrm{c}}=0.0536 C+1.32
$$

Where $\boldsymbol{C}$ is the velocity of wave in the solid polymer.

It should be noted that the relationship is linear in this case of the two-layer tubes with the above-defined dimensions.

\section{TIME-FREQUENCY ANALYSIS}

\subsection{Time-frequency images}

$S P W V$ given by the Eq. 6 is utilized in the work. Timefrequency images of the acoustic signals backscattered by the studied tubes are presented in Fig. 7. Reduced cutoff frequencies for $\boldsymbol{A}_{1}$ and $\boldsymbol{S}_{\boldsymbol{I}}$ pseudo-waves are estimated by the asymptotic lines along the time axis, and presented in the table 3.

Table 3. Estimated reduced cutoff frequencies from timefrequency images $\left(x_{1 c}\right)$

\begin{tabular}{|l|l|l|l|l|l|}
\hline Tube number & $\mathbf{0}$ & $\mathbf{1}$ & $\mathbf{2}$ & $\mathbf{3}$ & $\mathbf{4}$ \\
\hline $\boldsymbol{x}_{1 c}\left(\boldsymbol{A}_{1}\right)$ & 111 & 65.4 & 60.0 & 48.7 & 38.4 \\
\hline $\boldsymbol{x}_{I c}\left(\boldsymbol{S}_{\boldsymbol{I}}\right)$ & $\ldots$ & 134.7 & 119.4 & 98.7 & 82 \\
\hline
\end{tabular}

\subsection{Observation}

From time-frequency images, it seems that the $\boldsymbol{A}_{\boldsymbol{l}}$ and the $S_{I}$ waves results in the scattering process of guided waves propagating around the one-layer and the two-layer tubes. The reduced cutoff frequency values of $A_{I}$ and $S_{I}$ waves in the case of each tube, deduced from $W V$ and resonance trajectories, are comparable.

\section{CONCLUSION}

In the present paper, we have demonstrated that the study of acoustic signals backscattered by air-filled stainless steelsolid polymer two-layer tubes, immersed in water, reveals the effects of physical characteristics of the inner part on the guided waves propagation. The analysis of resonance trajectories and Wigner-Ville time-frequency images, toward normalized frequencies ranging from 0.1 to 200 , show the sliding of reduced cutoff frequencies of $A_{1}$ and $S_{1}$ guided waves towards low values. The relation between reduced cutoff frequency and velocity of wave in the solid polymer is linear. The process of repulsion phenomena of waves is manifested in the scattering phenomenon. More detailed work on this subject is also in progress. 

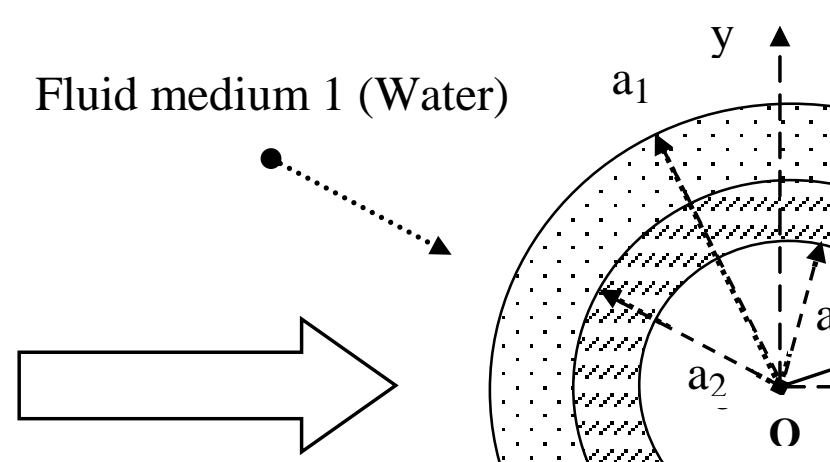

Incident plane wave
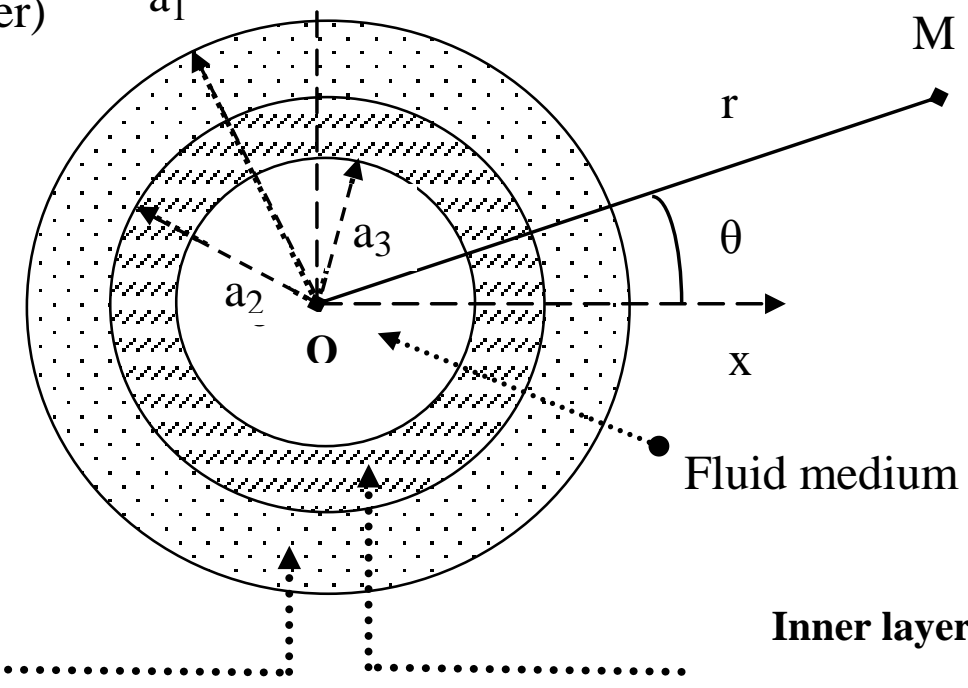

Fluid medium 2 (Air)

Outer layer

(Stainless Steel)
(Stainless Steel or Solid polymer)

Fig 1: Geometry of a plane wave normally incident on an air-filled cylindrical tube immersed in water
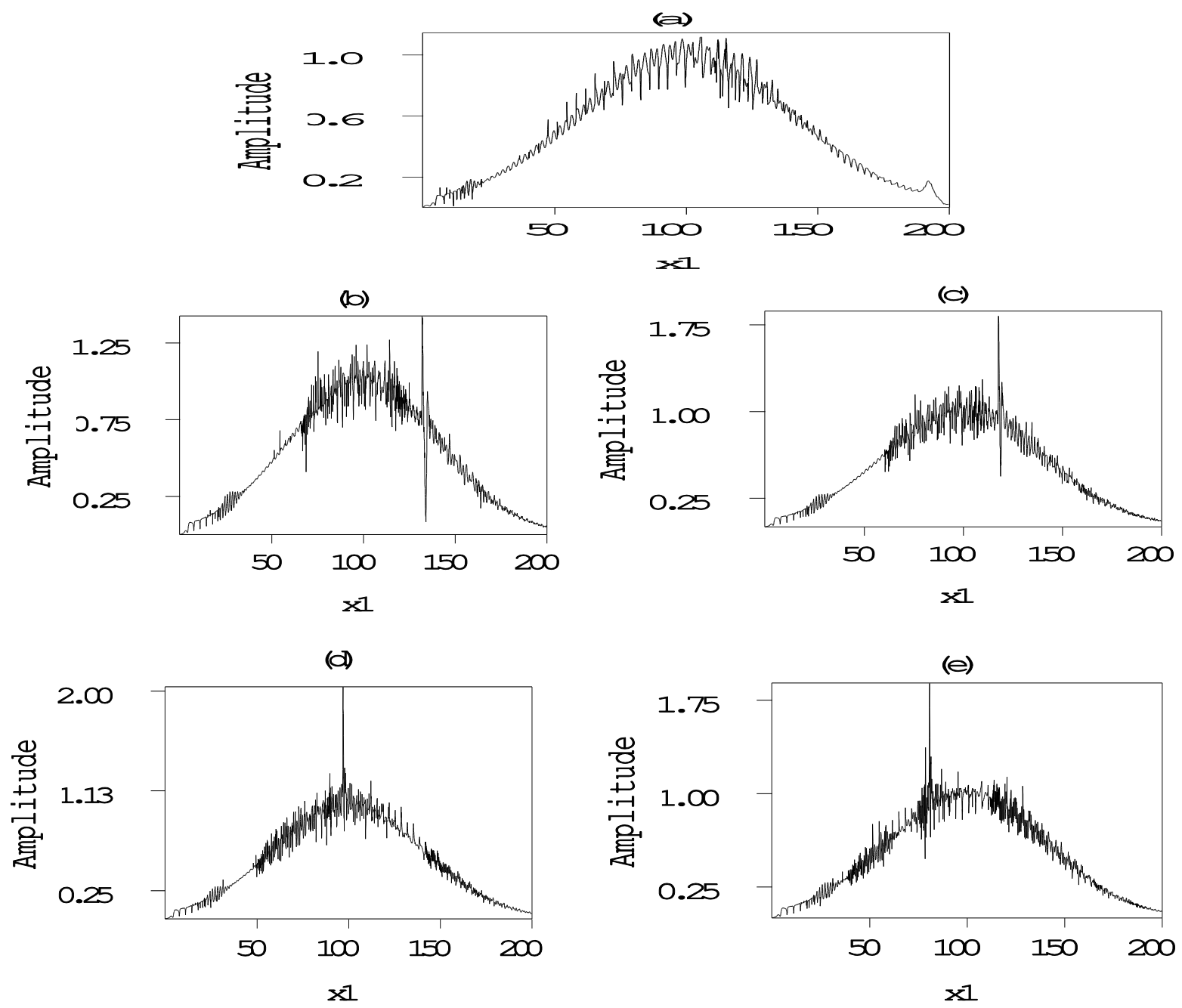

Fig 2: Calculated form functions of the Stainless Steel one-layer and the Stainless Steel - solid polymer two-layer tubes: (a) Tube 0 ; (b) Tube 1 ; (c) Tube 2 ; (d) Tube 3 ; (e) Tube 4. 

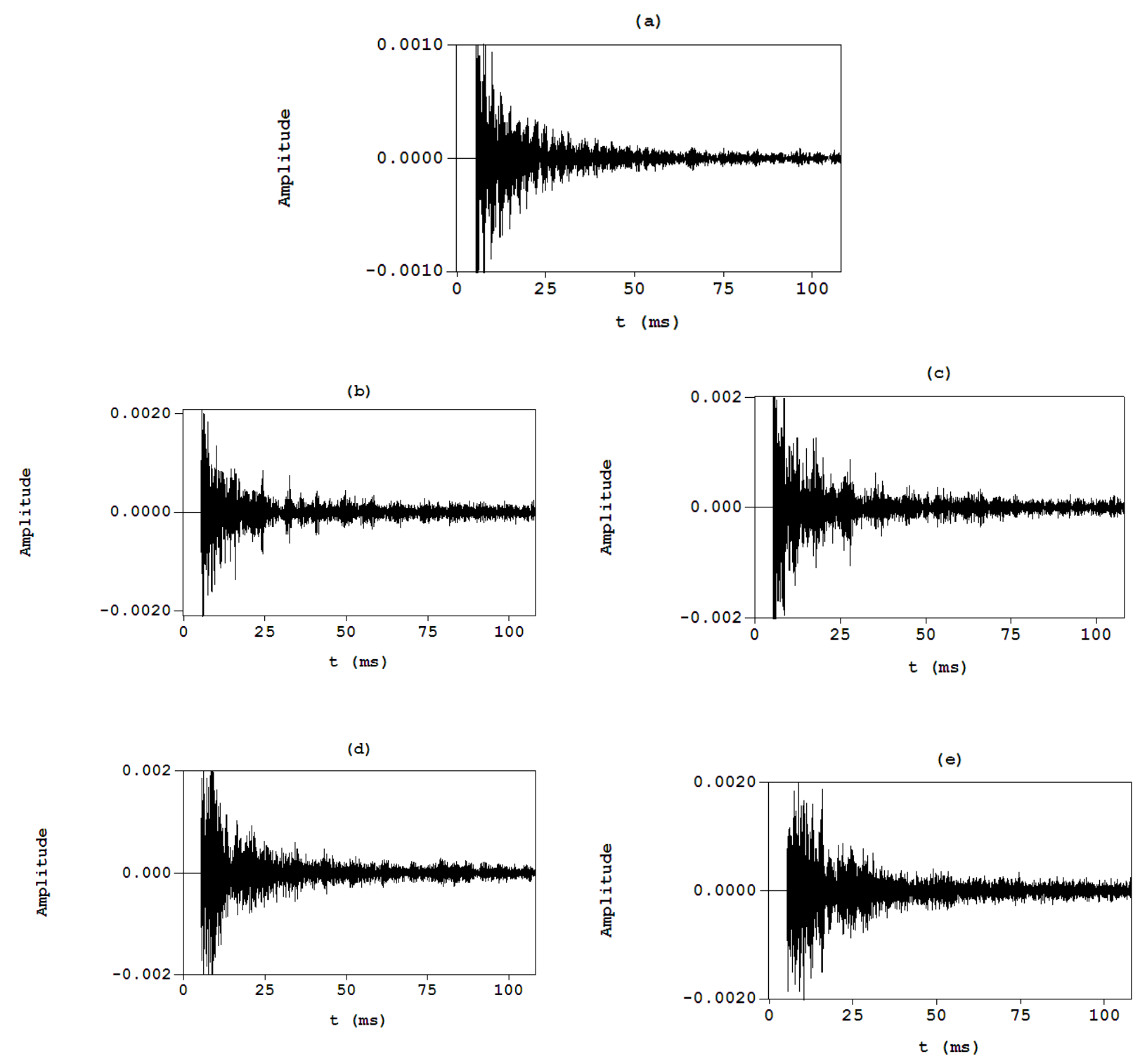

Fig 3: Time signals of the guided waves in: (a) Tube 0 ; (b) Tube 1 ; (c) Tube 2 ; (d) Tube 3 ; (e) Tube 4. 

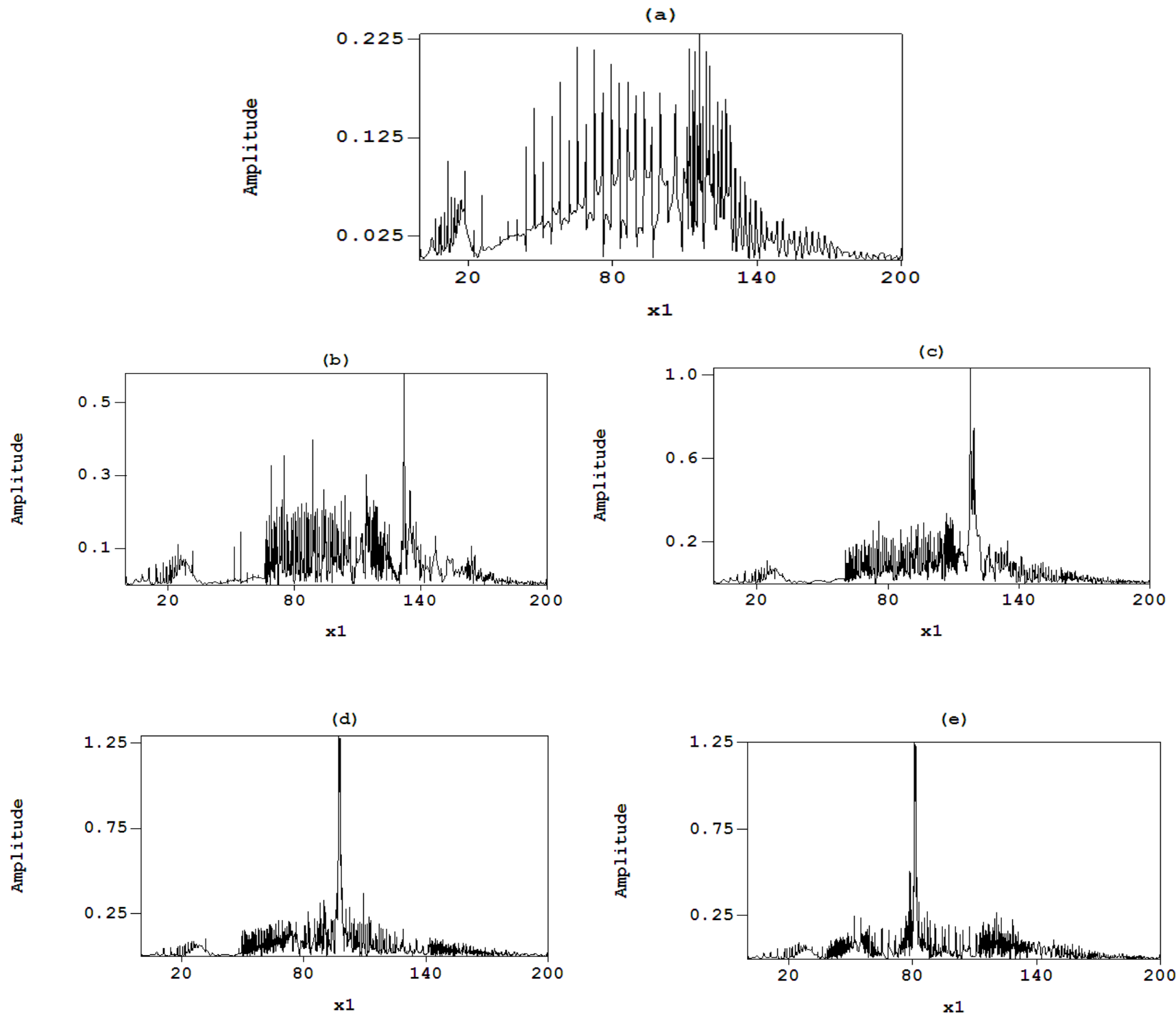

Fig 4: Resonance spectrums of the guided waves in : (a) Tube 0 ; (b) Tube 1 ; (c) Tube 2 ; (d) Tube 3 ; (e) Tube 4. 

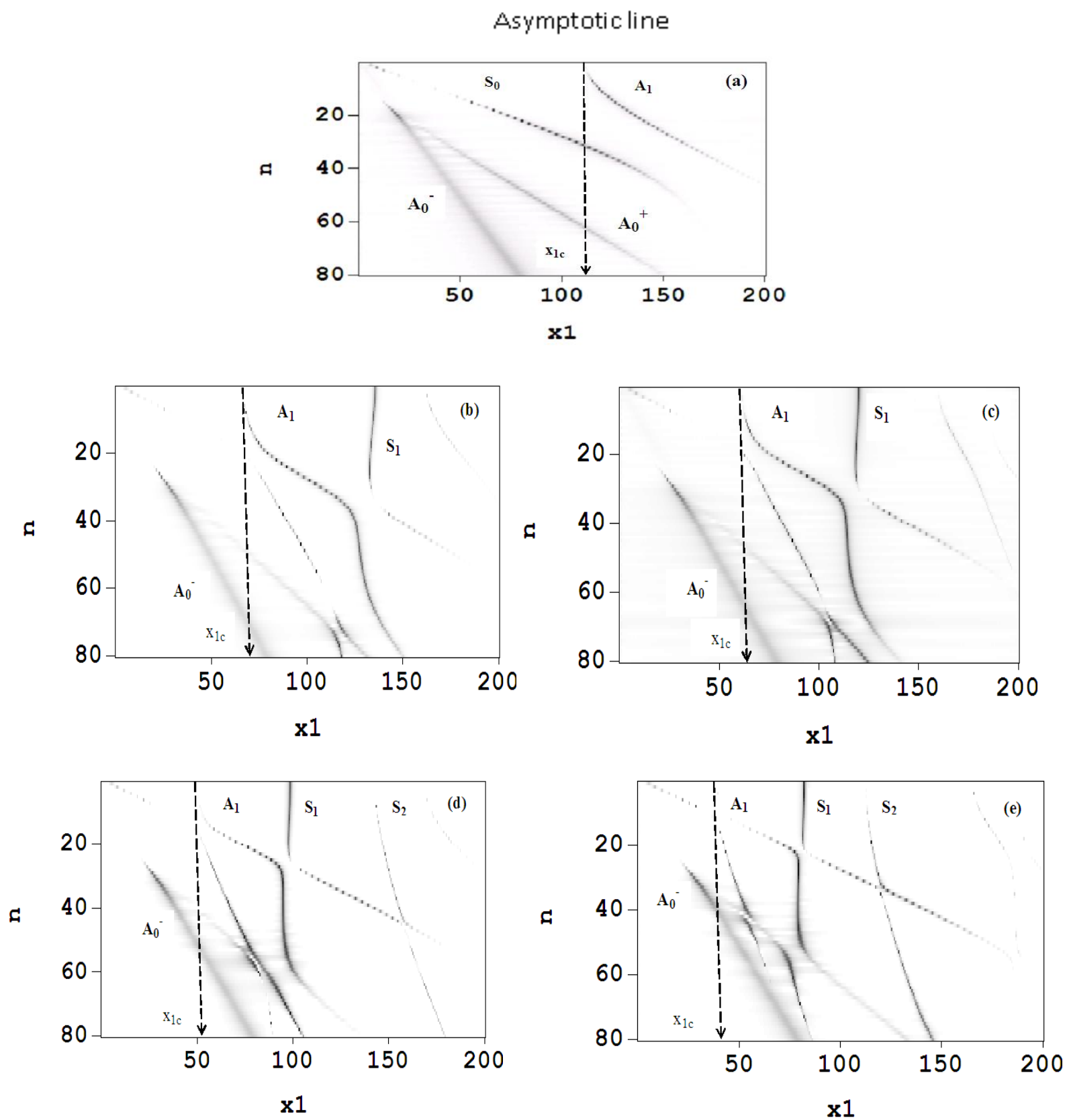

Fig 5: Resonance trajectories of the guided waves in: (a) Tube 0 ; (b) Tube 1 ; (c) Tube 2 ; (d) Tube 3 ; (e) Tube 4 


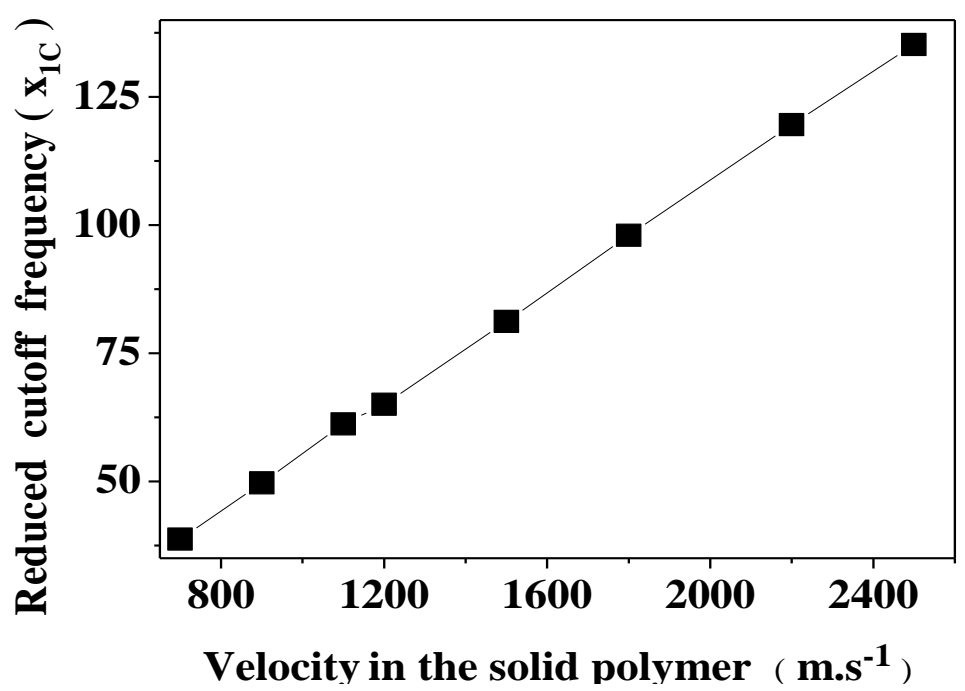

Fig 6: Visualization of reduced cutoff frequency of $A_{1}$ and $S_{1}$ as a function of velocity of wave in the solid polymer 

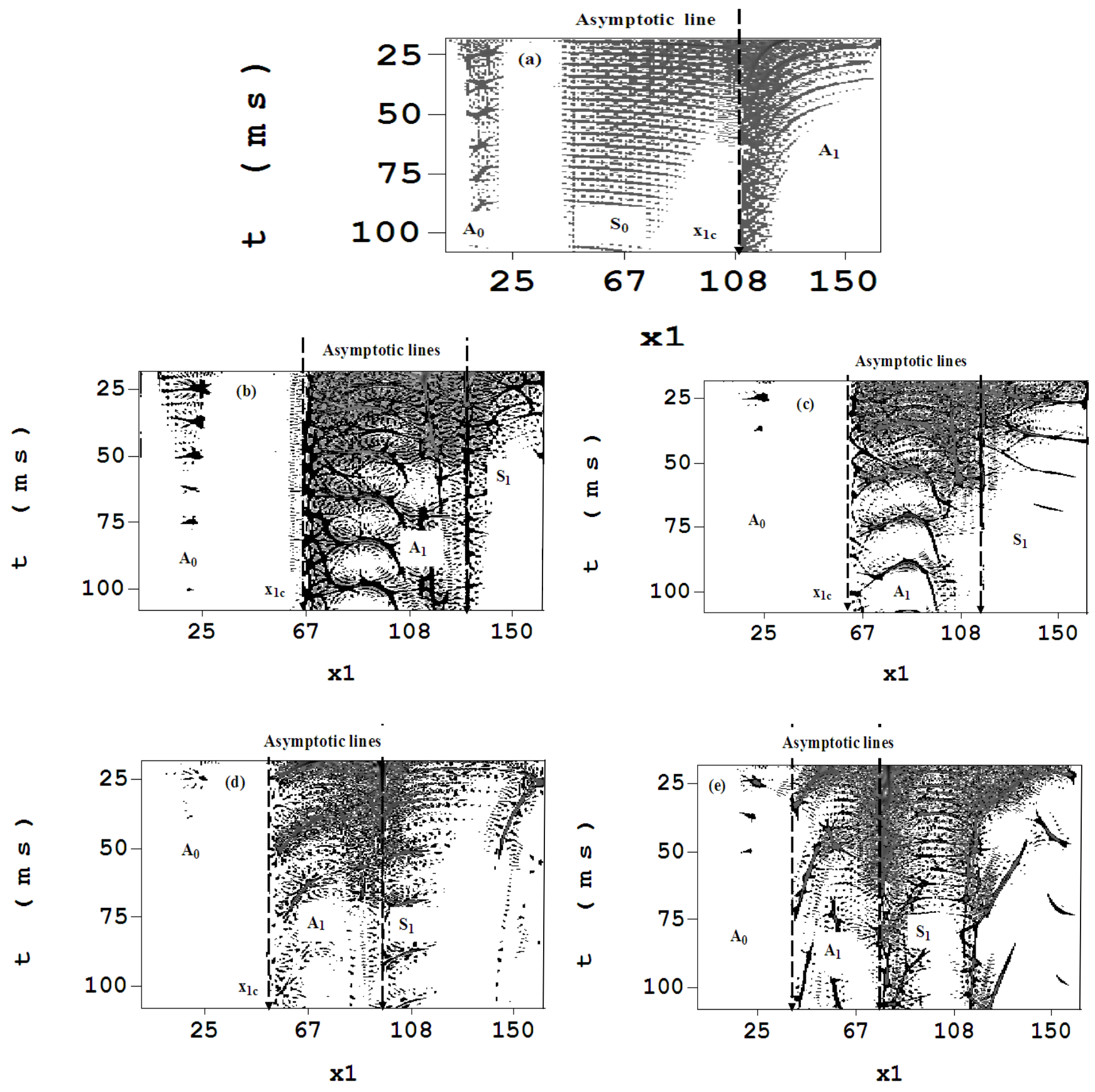

Fig 7: Wigner-Ville time-frequency images of the temporal signals in Fig. 3: (a) Tube 0 ; (b) Tube 1 ; (c) Tube 2 ; (d) Tube 3 ; (e) Tube 4

\section{REFERENCES}

[1] Murphy, J. D., Breitenbach, E. D. and Überall,H. 1978 Resonance scattering of acoustic waves from cylindrical shells, J. Acoust. Soc. Am. 64, 677-683.

[2] Flax, L., Gaunaurd, G. C. and Überall, H. 1981 Physical Acoustics XV, Academic Press, New York, 191-293.

[3] Maze, G., Ripoche, J. 1983 Méthode d'isolement et d'identification des résonances (M.I.I.R.) de cylindres et de tubes soumis à une onde acoustique plane dans l'eau, (Method of Isolation and Identification of Resonances (M.I.I.R.) of cylinders and cylindrical shells immersed in water), Revue de Physique Appliquée. 18, 319-326.
[4] Maze, G., Izbicki,J.-L. and Ripoche, J. 1985 Resonances of plates and cylinders: Guided waves, Journal of the Acoustical Society of America 77, 1352-1357.

[5] Sammelmann, G. S., Trivett, D. H., and Hackman, R. H. 1989 The acoustic scattering by a submerged, spherical shell. I. The bifurcation of the dispersion curve for the antisymmetric Lamb wave, J. Acoust. Soc. Am. 85, 114 124.

[6] Maze, G., Léon, F., Ripoche, J., Klauson, A., Metsaveer, J. and Überall, H. 1995 Nature de l'onde de Scholte sur une coque cylindrique, (Nature of the Scholte wave on a cylindrical shell), Acustica, International Journal on Acoustics 81, 201-213. 
[7] Maze, G., Léon, F., Ripoche, J. and Überall, H. 1999 Repulsion phenomena in the phase-velocity dispersion curves of circumferential waves on elastic cylindrical shells, Journal of the Acoustical Society of America, 105, 1695-1701.

[8] Maze, G., Izbicki, J.-L. and Ripoche,J. 1986 Acoustic scattering from cylindrical shells: guided waves and resonances of the liquid column, Ultrasonics 24, 354361.

[9] Chati, F., Léon, F. and Maze, G. 2005 Acoustic scattering by a metallic tube with a concentric solid polymer cylinder coupled by a thin water layer. Influence of the thickness of the water layer on the two ScholteStoneley waves, Journal of the Acoustical Society of America 118, 2820-2828.

[10] Liang-Wu Cai, and Sànchez Dehesa, J. 2008 Acoustic scattering by radially stratified scatterers, Journal of the Acoustical Society of America 124, 2715-2726.

[11] Hashminejad, S. M. and Rajabi, M. 2008 Acoustic scattering from functionally graded cylindrical shells, Journal Sound and Vibration 202, 208-228.

[12] Jamali, J., Naet, M. H., Honarvar, F. and Rajabi, M. 2011 Acoustic scattering from functionally graded cylindrical shells, Arch. Mech. Warszawa, 63 (1), 25-56.
[13] Jiangong Yu, Lefebvre, J. E. and Elmaimouni, L. 2013 Toroidal wave in multilayered spherical curved plates, Journal Sound and Vibration 332, 2816-2830.

[14] Hasheminejad, S.M. and Safari, N. 2005 Acoustic scattering from viscoelastically coated spheres and cylinders in viscous fluids, Journal of Sound and Vibration 280, 101-125.

[15] Flax, L., Varadan, V. K. and Varadan, V. V. 1980 Scattering of an obliquely incident wave by an infinite cylinder, J. Acoust. Soc. Am. 68, 1832-1835.

[16] Abramovitz, M. and Stegun, I.A. 1964 Handbook of Mathematical Functions, National Bureau of Standards, Washington, DC, 435-442.

[17] Flax, L., Gaunaurd, G. C. and Überall, H. 1981 Theory of Resonance Scattering, in Vol. 15 of Physical Acoustics Academic, New York, Chap. 3, 191-294.

[18] Cohen, L. 1995 Time-Frequency Analysis, Prentice Hall, PTR, the City University of New York, 1-316.

[19] Cohen, L. 1989 Time-frequency distribution - a review, Proc IEEE, 77(7), 941-981.

[20] Flandrin, P. 1993 Temps-Fréquence, Hermès, Paris, 7 390. 